\title{
Data Visualization on Mobile Devices
}

Bongshin Lee

Matthew Brehmer

Microsoft Research

Redmond, WA 98052, USA

bongshin@microsoft.com

mabrehme@microsoft.com
Eun Kyoung Choe

University of Maryland

College Park, MD 20742, USA

choe@umd.edu

\author{
Petra Isenberg \\ Inria \\ 91405 Orsay, France \\ petra.isenberg@inria.fr
}

Ricardo Langner

Raimund Dachselt

Technische Universität Dresden

01062 Dresden, Germany

langner@acm.org

dachselt@acm.org

Permission to make digital or hard copies of part or all of this work for personal or classroom use is granted without fee provided that copies are not made or distributed for profit or commercial advantage and that copies bear this notice and the full citation on the first page. Copyrights for third-party components of this work must be honored. For all other uses, contact the owner/author(s).

Copyright held by the owner/author(s).

CH'18 Extended Abstracts, April 21-26, 2018, Montreal, QC, Canada ACM 978-1-4503-5621-3/18/04.

https://doi.org/10.1145/3170427.3170631

\begin{abstract}
As mobile visualization is increasingly used and new mobile device form factors and hardware capabilities continuously emerge, it is timely to reflect on what has been discovered to date and to look into the future. This workshop will bring together researchers, designers, and practitioners from relevant application and research fields, including visualization, personal informatics, and data journalism. We will work on identifying a research agenda for mobile data visualization as well as to collect and propagate practical guidance for mobile visualization design. Our overarching goal is to bring us closer to making an effective use of ubiquitous mobile devices as data visualization platforms.
\end{abstract}

\section{Author Keywords}

Data visualization; interaction; mobile devices; mobile visualization; visualization design.

\section{ACM Classification Keywords}

H.5.m [Information interfaces and presentation (e.g., $\mathrm{HCl}$ )]: Miscellaneous.

\section{Introduction}

Despite recent advances in pen- and/or touch-enabled mobile devices and the rapid adoption of these devices in everyday life, we are far from leveraging the full potential of mobile devices in satisfying the growing demand for visual 


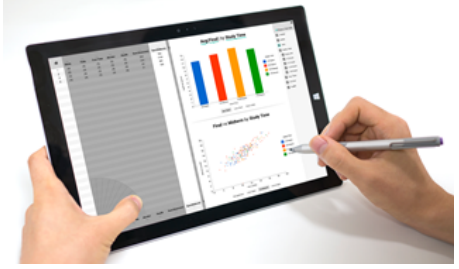

Figure 1: TouchPivot [7] supports data exploration on tablets.

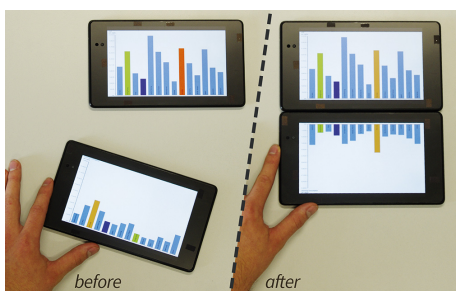

Figure 2: VisTiles [11] leverages multiple mobile devices for spatially-aware exploration of multivariate data.

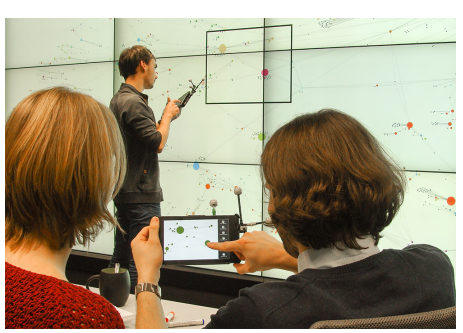

Figure 3: GraSp [10] allows the collaborative use of multiple mobile devices for graph visualization. access to data. Even though the design space for mobile data visualization is emerging out of everyday practice [14], concentrated research efforts have not yet emerged.

Two curated collections of mobile visualization ${ }^{1,2}$ showcase examples from the practitioner community, illustrating the range of visual encoding choices and design patterns in use. Such collections are helpful resources, as applications for monitoring personal health, finance, and travel data are now designed solely for mobile use, or at least with a mobile-first mentality. Similarly, most large journalism outlets now prioritize their mobile readership [1], and news articles containing static or interactive visualization should comply with this mentality. However, usage examples alone are not enough to determine how to design better data visualization tools for mobile devices.

Researchers across several HCl-related communities thus have investigated this question. For example, some visualization research addresses visual encoding choices, such as the challenge of encoding temporal uncertainty on small devices [8]. Others have explored the space of touch interactions for popular chart types [2, 5, 6, 13] and data exploration on tablets (Fig. 1) [7], and ways to leverage the position and orientation of mobile devices to support the exploration of 3D representations of data [3]. Another interesting recent research direction examines opportunities for combining multiple mobile devices as spatially distributed, tangible visualization views (Fig. 2) [11] or in combination with large display walls (Fig. 3) [10].

Mobile self-tracking and data collection apps increasingly use data visualization to enable people to visually access their data without relying on a desktop or laptop. Recent

$$
\begin{aligned}
& { }^{1} \mathrm{http}: / / \text { mobilev.is } \\
& { }^{2} \mathrm{http}: / / \text { mobileinfovis.com }
\end{aligned}
$$

mobile apps emanating from the research community include SleepTight, which visualizes sleep patterns in terms of sleep duration and quality for a given time frame (e.g., last week) on a mobile device [4]. Another example is ConCap, a mobile app that enables diabetic patients to collect and display their data along a timeline, allowing them to reflect on and manage their chronic conditions [12]. In addition, OmniTrack employs a visualization dashboard featuring different charts for the various data types that the app collects [9]. With regards to commercial mobile apps, most self-tracking devices such as activity trackers (e.g., Fitbit, Misfit) and blood pressure monitors (e.g., Omron, Nokia $\mathrm{BPM}+$ ) have associated mobile apps that leverage data visualization. However, despite the increased prevalence of visualization on mobile devices, we are missing a consolidated set of best practices and ways to evaluate mobile data visualization.

In summary, both mobile visualization researchers and practitioners are tackling shared problems, such as how to adapt and optimize data representations to small displays; how to support novel and effective interactions with small data representations; and how to best use mobile devices in a device ecosystem and with multiple collaborators. Furthermore, new challenges continue to emerge as data visualization techniques are adopted and used for novel mobile apps. In addition, new mobile device form factors and hardware capabilities will emerge in the coming years. Thus, it is timely to take stock of what has been discovered to date and to look to the future. This workshop will bring together researchers and practitioners from relevant application and research fields, including visualization, mobile interaction design, personal health, emergency response, and journalism, in an effort to set a research agenda for mobile data visualization and to collect and propagate actionable guidance for mobile app developers. 


\section{Goals}

This workshop is intended to be a platform to exchange information and experiences, to stimulate discussion, and to identify novel aspects and ideas around mobile data visualization. While the visualization research community has over the years developed a large set of recommendations for visualization development on a variety of devices, how these recommendations scale (down) to mobile devices is under-explored. Practitioners largely rely on their design intuition to solve known challenges such as labeling \& color coding for small data items, fat finger problems, low data \& visualization literacy among their target audience, and the absence of mouse-over interaction, which is prevalent in desktop-based interactive visualization systems.

An immediate goal of this workshop is thus to identify open problems that need further investigation and to identify priorities for future research in this space. Furthermore, one of the overarching goals of this workshop is to bring participants with diverse backgrounds together to identify the most pressing mobile visualization research challenges that evolve out of practice and to establish opportunities for future collaboration. We plan to solicit involvement from multiple application domains so that we can effectively synthesize and share best practices from a variety of domainspecific challenges.

During our workshop we would like to put an emphasis on the following three questions:

1. What are the mobile-inherent and application-specific challenges in designing interfaces for visualizing and exploring data on mobile devices?

2. How can we address these challenges?

3. What are new and exciting ways to use mobile devices for data visualization?

\section{Scopes and Topics}

We solicit various types of workshop contributions on visualizing data on a wide range of mobile devices, including tablets, smart phones, smart watches, wearable devices (with screens), and tiny screens (e.g., fingernail displays):

Research papers that describe existing data visualization on mobile devices, novel interface approaches \& interaction techniques, or early concepts in the form of design vignettes, pictorials, or sketches;

Position papers, commentaries, and critiques of existing designs or of design and evaluation processes;

Demonstrations of prototypes and completed projects.

With the help of an international program committee we will select submissions based on a number of criteria, including originality or controversy of the paper, the complementary nature of the authors' research background, and the quality of the authors' previous work related to the workshop topic.

The topics we will cover during the workshop include (are not limited to):

- Visual encodings

- Personal data visualization

- Glanceable visualization

- Responsive visualization

- Data-driven storytelling

- Natural interaction techniques

- Multimodal and multi-device interaction

- Visualization tasks

- Visualization authoring

- Libraries and toolkits

- Evaluation methods

- Best practices and pitfalls in the design process 
We hope to develop each of these topics further through the solicitation of the workshop papers and via workshop activities including paper presentations, discussions, and a consolidation of best practices.

\section{Pre-workshop Plans}

Research relating to data visualization on mobile devices has appeared in various $\mathrm{HCl}$ subcommunities including ACM conferences such as CHI, MobileHCl, ISS, UbiComp, and UIST, as well as IEEE conferences such as InfoVis and VAST. We therefore intend to spread our call for participation across these communities.

With respect to involving practitioners in this workshop, we will draw upon our connections with developers of mobile personal health apps at organizations such as Microsoft, Google, and Fitbit, several of whom regularly attend $\mathrm{CHI}$. We will also draw upon our connections with mobile app developers, digital media organizations, freelance visualization designers, and news graphics designers who have published mobile-first visualization projects.

In our workshop website (mobilevis. github.io), we posted our call for participation and introduced the workshop organizers. Once we have a list of accepted papers, we will share them through the workshop website; we will also ask workshop participants to upload workshop-related information such as a brief bio and profile picture, their goals and interests, and an image representative of their work. In addition, for effective communication with authors of the accepted papers, we will invite workshop participants to a workspace on Slack (mobilevis.slack.com).

\section{Workshop Structure}

The focus of the workshop will be an interactive dialog up to 25 attendees (including organizers) with multidisciplinary backgrounds. The first part of the workshop provides the opportunity for participants to introduce their projects, research, and perspectives. The second part incorporates break-out sessions with hands-on design activities using paper and other traditional crafting material.

Table 1 shows a preliminary schedule on how our workshop will be organized. It is important for us to ensure that the workshop allows attendees to have a holistic experience of the overall research topic ranging from technical solutions used in specific projects to future visions.

After an opening, the morning session allows all participants to briefly introduce themselves by means of short paper and position statement presentations. To avoid the semblance of a small conference, we encourage short presentations (of about five minutes) for individual contributions. Depending on the number of submissions, we plan to have 2-4 thematically grouped presentation sessions in the morning. Each round will end with a short wrap-up discussion to seek a common ground for afternoon discussions.

These morning session also allows time for invited talks by selected experts from research communities and industry.

\begin{tabular}{l|l|l}
$\mathbf{0 9 . 0 0}-\mathbf{0 9 . 1 5}$ & Opening and introduction & $15 \mathrm{~min}$. \\
$\mathbf{0 9 . 1 5}-\mathbf{1 0 . 4 5}$ & Participants' Presentations I, II & $90 \mathrm{~min}$. \\
$\mathbf{1 0 . 4 5}-\mathbf{1 1 . 1 5}$ & Coffee break & $30 \mathrm{~min}$. \\
$\mathbf{1 1 . 1 5}-\mathbf{1 2 . 4 5}$ & Participants' Presentations III, IV & $90 \mathrm{~min}$. \\
$\mathbf{1 2 . 4 5}-\mathbf{1 4 . 0 0}$ & Lunch & $75 \mathrm{~min}$. \\
$\mathbf{1 4 . 0 0 - 1 4 . 4 5}$ & Demos and hands-on experiences & $45 \mathrm{~min}$. \\
$\mathbf{1 4 . 4 5}-\mathbf{1 5 . 4 5}$ & Envisioning \& design (group work) & $60 \mathrm{~min}$. \\
$\mathbf{1 5 . 4 5 - 1 6 . 1 5}$ & Coffee break & $30 \mathrm{~min}$. \\
$\mathbf{1 6 . 1 5}-\mathbf{1 6 . 4 5}$ & Group results \& reflection & $30 \mathrm{~min}$. \\
$\mathbf{1 6 . 4 5} \mathbf{- 1 7 . 4 5}$ & Breakout session \& brainstorming & $60 \mathrm{~min}$. \\
$\mathbf{1 7 . 4 5 - 1 8 . 0 0}$ & Workshop wrap-up and closing & $15 \mathrm{~min}$.
\end{tabular}

Table 1: Preliminary workshop schedule. 
After a joint lunch, which allows participants to meet and network informally, the workshop will continue with handson experiences in a relaxed demo atmosphere. Short demonstrations of different forms of prototypes, including sketches, case studies, and implementations (solicited from workshop participants and industry), will foster a discussion of stateof-the-art design and implementation approaches.

The afternoon session will continue with a creative envisioning \& design session, during which small groups of 3-5 people will work on specific ideas for future mobile visualization concepts. To support creativity and originality, we will make use of paper prototyping and crafting materials. After the afternoon coffee break, each group will pitch their project (such as by acting out scenarios), and we will quickly discuss the potential of each concept following each pitch. In a final break-out and brainstorming session, participants will collect ideas, concepts, and issues on the future of data visualization on mobile devices. Written on cards or notes, we will try to group and discuss ideas for shaping a research agenda in this emerging field.

The official part of the workshop will conclude with a brief summary discussion. We plan to organize a joint dinner to further socialize and continue discussions and networking.

\section{Post-workshop Plans}

We will summarize the workshop outcomes including a research agenda and a set of design guidelines that emerge from the workshop discussions and activities, and we will share the summary and all presented materials on our website. In addition, we intend to submit a research paper distilling research-specific content from the workshop in a journal or magazine. We will also consider editing a special journal issue on the topic of mobile data visualization to further raise the interest in this exciting research area.
In addition to the research outcomes, we will grow our website after the workshop to become a new repository of mobile visualization by showcasing examples from workshop participants and by continually adding additional resources and designs. We will continue our discussion and communication with the workshop participants through the MobileVis Slack channel. More importantly, we intend to organize a Dagstuhl seminar in the near future to foster further collaboration, enlarge the community, and progress the topic of mobile data visualization in a significant effort.

\section{Organizers}

Our organization team is comprised of members having several relevant qualifications that will ensure a successful workshop. The core background of our team members range from visualization research (Brehmer, Isenberg, \& Lee) to $\mathrm{HCl}$ (Choe, Dachselt, Langner, \& Lee) to UbiComp (Choe). All team members have previously organized industry and academic workshops in several venues, and have extensive experience in reviewing publications for international conferences, workshops, and symposia.

Bongshin Lee is a Senior Researcher at Microsoft Research. She explores innovative ways to enable people to create visualizations, interact with their data, and share data-driven stories. She has been recently focusing on helping people collect \& explore the data about themselves, and share insights with others by leveraging visualizations. She earned her Ph.D. in Computer Science from University of Maryland at College Park in 2006.

Matthew Brehmer is a Postdoctoral Researcher at Microsoft Research and a representative of the Microsoft Data Journalism Program. His research interests involve designing and evaluating information visualization techniques for storytelling and journalism, considering the perspectives 
of both the audience or information consumer and the author or content creator. He is the co-chair of the 2018 IEEE PacificVis Visual Data Storytelling Contest and the Visualization in Practice event at IEEE VIS 2018. He received his Ph.D. in Computer Science from the University of British Columbia in Vancouver in 2016.

Petra Isenberg is a Research Scientist at Inria. Her main research areas are information visualization and visual analytics with a focus on collaborative work scenarios, interaction, and evaluation. She is interested in exploring how people can most effectively analyze data sets on novel display technology such as small touch-screens, wall displays, or tabletops. She received her Ph.D. in Computer Science from the University of Calgary in 2009.

Eun Kyoung Choe is an Assistant Professor in the College of Information Studies at University of Maryland at College Park. Her areas of research include $\mathrm{HCl}$, Health Informatics, and UbiComp. She has been examining ways to help people become empowered individuals through fully leveraging their personal data in various contexts including the Quantified Self movement, sleep, patient-clinician communication, and personal data insights \& visualization. She received her Ph.D. in Information Science from University of Washington in 2014.

Ricardo Langner is a Ph.D. student at the Technische Universität Dresden. His research focuses on the combination of multiple displays, such as mobile devices, interactive tabletops, and large shared displays, for information visualization. In particular, he explores innovative ways of using mobile devices to support data exploration with multiple coordinated visualization views. He was also a local arrangements co-chair of ACM ITS 2014.
Raimund Dachselt is a full university professor for Computer Science at Technische Universität Dresden and a director of the Interactive Media Lab Dresden. His research focuses on Natural User Interface (NUI) approaches with various input modalities on interactive surfaces and in multidisplay environments. His team contributed several solutions applying NUI techniques to the field of Information Visualization and Immersive Analytics. He received his Ph.D. in Computer Science from TU Dresden in 2004.

\section{Acknowledgment}

This work was in part supported by the National Science Foundation under award number CHS-1753425.

\section{REFERENCES}

1. Gregor Aisch. 2016. Data Visualization and the News. (2016). https://vimeo.com/182590214 Invited talk at the Information+ Conference.

2. Dominikus Baur, Bongshin Lee, and Sheelagh Carpendale. 2012. TouchWave: Kinetic Multi-touch Manipulation for Hierarchical Stacked Graphs. In Proceedings of the ACM International Conference on Interactive Tabletops and Surfaces (ITS). 255-264. DOI : http: //dx.doi.org/10.1145/2396636. 2396675

3. Wolfgang Büschel, Patrick Reipschläger, Ricardo Langner, and Raimund Dachselt. 2017. Investigating the Use of Spatial Interaction for 3D Data Visualization on Mobile Devices. In Proceedings of the ACM Conference on Interactive Surfaces and Spaces (ISS). 62-71. DOI :

http://dx.doi.org/10.1145/3132272.3134125

4. Eun Kyoung Choe, Bongshin Lee, Matthew Kay, Wanda Pratt, and Julie A. Kientz. 2015. SleepTight: Low-burden, Self-monitoring Technology for Capturing and Reflecting on Sleep Behaviors. In Proceedings of 
the ACM International Joint Conference on Pervasive and Ubiquitous Computing (UbiComp). 121-132. DOI : http://dx.doi.org/10.1145/2750858.2804266

5. Steven M. Drucker, Danyel Fisher, Ramik Sadana, Jessica Herron, and m.c. schraefel. 2013. TouchViz: A Case Study Comparing Two Interfaces for Data Analytics on Tablets. In Proceedings of the ACM SIGCHI Conference on Human Factors in Computing Systems (CHI). 2301-2310. DOI :

http://dx.doi.org/10.1145/2470654.2481318

6. Jaemin Jo, Bongshin Lee, and Jinwook Seo. 2015. WordlePlus: expanding wordle's use through natural interaction and animation. IEEE computer graphics and applications 35, 6 (2015), 20-28.

7. Jaemin Jo, Sehi L'Yi, Bongshin Lee, and Jinwook Seo. 2017. TouchPivot: Blending WIMP \& Post-WIMP Interfaces for Data Exploration on Tablet Devices. In Proceedings of the $2017 \mathrm{CHI}$ Conference on Human Factors in Computing Systems. ACM, 2660-2671.

8. Matthew Kay, Tara Kola, Jessica R. Hullman, and Sean A. Munson. 2016. When (Ish) is My Bus? User-centered Visualizations of Uncertainty in Everyday, Mobile Predictive Systems. In Proceedings of the ACM SIGCHI Conference on Human Factors in Computing Systems (CHI). 5092-5103. DOI : http://dx.doi.org/10.1145/2858036.2858558

9. Young-Ho Kim, Jae Ho Jeon, Bongshin Lee, Eun Kyoung Choe, and Jinwook Seo. 2017. OmniTrack: A Flexible Self-Tracking Approach Leveraging Semi-Automated Tracking. Proceedings of the ACM Journal on Interactive, Mobile, Wearable and Ubiquitous Technologies 1, 3 (2017), 67:1-67:28. DOI : http://dx.doi.org/10.1145/3130930
10. Ulrike Kister, Konstantin Klamka, Christian Tominski, and Raimund Dachselt. 2017. GraSp: Combining Spatially-aware Mobile Devices and a Display Wall for Graph Visualization and Interaction. Computer Graphics Forum 36, 3 (2017), 503-514. DOI : http://dx.doi.org/10.1111/cgf.13206

11. Ricardo Langner, Tom Horak, and Raimund Dachselt. 2018. VisTiles: Coordinating and Combining Co-located Mobile Devices for Visual Data Exploration. IEEE Transactions on Visualization and Computer Graphics 24, 1 (2018). DOI :

http://dx.doi.org/10.1109/TVCG.2017.2744019 In press.

12. Tom Owen, Jennifer Pearson, Harold Thimbleby, and George Buchanan. 2015. ConCap: Designing to Empower Individual Reflection on Chronic Conditions using Mobile Apps. In Proceedings of the 17th International Conference on Human-Computer Interaction with Mobile Devices and Services. ACM, 105-114.

13. Ramik Sadana and John Stasko. 2016. Expanding Selection for Information Visualization Systems on Tablet Devices. In Proceedings of the ACM Conference on Interactive Surfaces and Spaces (ISS). 149-158. DOI :http://dx.doi.org/10.1145/2992154. 2992157

14. Benjamin Watson and Vidya Setlur. 2015. Emerging Research in Mobile Visualization. In Tutorial Proceedings of the International Conference on Human-Computer Interaction with Mobile Devices and Services (MobileHCl). 883-887. DOI :

http://dx.doi.org/10.1145/2786567.2786571 


\section{Appendix: Call for Participation}

With an increasing use of mobile devices in everyday life, it is important to understand how to design data visualizations for small displays. A nuanced understanding is especially important since existing guidelines for visualization and data interaction design do not transfer well to mobile devices. This one-day workshop aims to bring together visualization researchers and practitioners from application domains, to share experiences, identify open problems, and explore the future of mobile visualization. We will share academic and application-related outputs, and conduct hands-on design and brainstorming activities.

We solicit 4-6 page workshop papers excluding references (CHI Extended Abstracts format) in the following contribution types:

Research papers describing existing mobile data visualizations or early concepts;

Position papers discussing existing \& future designs and processes;

Demonstrations of prototypes and completed projects.

Submissions should cover contributions on visualizing data on various mobile devices (e.g., tablets, smart phones/watches, wearable devices, and tiny screens) addressing the following topics:

- Visual encodings

- Personal data visualization

- Glanceable visualization

- Responsive visualization

- Data-driven storytelling

- Natural and multi-modal interaction

- Visualization tasks

- Visualization authoring

- Libraries and toolkits

- Evaluation methods

- Best practices and pitfalls in the design process

Please submit a paper to the EasyChair system (https://easychair.org/conferences/?conf=mobilevis2018). We will select submissions based on a number of criteria, including originality or controversy of the paper, the complementary nature of the authors' research background, and the quality of the authors' previous work related to the workshop topic.

At least one author of each accepted paper must attend the workshop and all workshop participants must register not only for the workshop but also for at least one day of the conference.

Workshop information: mobilevis.github.io | Contact: mobilevisualization@gmail.com | Twitter: @mobilevis 